\title{
KEY TECHNOLOGIES IN WSN-BASED COTTON FIELD SOIL MOISTURE MONITORING SYSTEM
}

\author{
Ruirui Zhang ${ }^{1}$, Liping Chen ${ }^{1, *}$, Gang Xu ${ }^{1}$, Yanji Wang ${ }^{1}$ \\ ${ }^{1}$ National Engineering Research Center for Information Technology in Agriculture, Beijing, \\ P. R. China 100089 \\ * Corresponding author Address: Room A517, Beijing Nongke Building, No.11, \\ Shuguanghuayuan Mid-road, Haidian District, Beijing,P.R.China,100089 Tel: +86-10- \\ 51503425, Fax: +86-01-51503425, Email: chenlp@nercita.org.cn
}

Abstract: To level up the application of wireless sensor network in cotton field soil monitoring, wireless sensor network technology has been studied deeply. Some difficulties, including the design of soil moisture sensor, covering problem in wireless sensor network and diagnosis algorithm for wireless sensor networks and node, are been proposed and discussed. Combined with cotton field soil moisture monitoring application, coverage and deployment based not only on plane space but also on time are been discussed and a fault diagnosis algorithm based on entropy is been put forward in the article.

Key words: wireless sensor network, field soil moisture monitor, coverage algorithm, fault diagnosis algorithms

\section{INTRODUCTION}

Precision agriculture (PA) is an agricultural concept relying on the existence of in-field variability. It's about doing the right thing, in the right place, in the right way, at the right time. It's connotation is regulate the crop inputs according to the current soil properties, which will reach the goal of optimizing formula, scientific management, activating the soil production so that we can get equal or high production by the same input. PA is the

Please use the following format when citing this chapter:

Zhang, R., Chen, L., Xu, G. and Wang, Y., 2009, in IFIP International Federation for Information Processing, Volume 295, Computer and Computing Technologies in Agriculture II, Volume 3, eds. D. Li, Z. Chunjiang, (Boston: Springer), pp. 2133-2140. 
direction of future agriculture and can get the result of improving environment. Acquirement of the soil parameter is an important part in PA.

Soil moisture is an important factor to cotton plant, which will influence its quality and quantity. Insufficiency of water leads to thirsty of cotton plant, and overabundance of water results in not only the waste of water but also shallow root and weak ability for anti-lodging. Getting the current cottonfield soil moisture content precisely and irrigating the field quantitatively and distinctively become an urgent problem to solve.

Research in existing technology of soil moisture monitoring reveals that precise and prompt result can not be got properly. There are mainly two ways to get soil moisture content. The first is getting soil sample manually, then after firing and weighing, to get the field soil moisture result. This method requires a lot of manpower and lack real-time property, which can not satisfy the demand of PA obviously. The second popular way is via the WLAN (Wireless local area network, WLAN) technology. But there are also some shortcomings of the way. High power consumption of WLAN module and monotonous topology of network restrict the geographical distribution of sample point, which can lead to inaccuracy result. In light of the above situation, system of WSN-based cotton field moisture monitoring is proposed.

\section{WIRELESS SENSOR NETWORK}

Wireless sensor network(WSN) considered as one of the most affected technology to people's daily life in $21^{\text {st }}$ century, is a wireless network consisting of spatially distributed autonomous devices using sensors to monitor physical or environmental conditions cooperatively at different locations. (Jing Qi et al., 2007) Comparing to traditional technique, the WSNbased monitoring system has following advantages:

a) Low impact on field environment. For one thing, each sensor node is very small, so being placed in field hardly have affect to work of farming machinery. For another, nodes are powered by AA alkaline battery and communicate with each other by radio, so that nodes can be placed according to our need and free from cable. Up to some extent, WSN-based farmland moisture monitoring system has the least impact on field local environment.

b)High dense sampling point. As the nodes are tiny and can relay message each other, they can be placed at high density. By this, more accurate soil moisture content can be got.

c) Low cost comparing with manual sampling and WLAN- based way. 


\section{SYSTEM FRAME AND KEY TECHNICAL DIFFICULTIES}

\subsection{System framework}

Work principle of the monitoring system: sensor nodes, equipped with RF communication module and distributed in a cotton field, sense the cottonfield soil moisture content and send data in relay through the radio wave from a node to another. At the end, all the sensor data is collected and swarms into a WSN-GPRS gateway which processes the data package, and then retransmits the data to data management center through the GPRS network. Data management center provides decision reference for irrigation after analyzing the data. People can get access to the decision reference message by an Internet-connected PC or a PDA. Figure 1 shows the system concrete construction. (Chen et al., 2004)

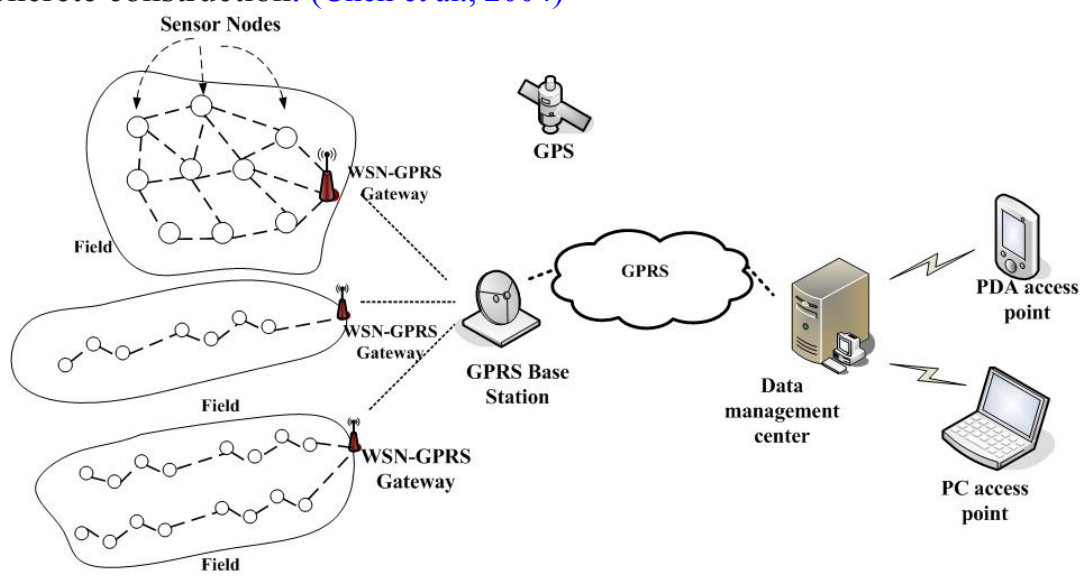

Figure 1 Scheme of the WSN structure

In the application of cotton field, mainly three aspects of technology need to study: soil moisture sensor, coverage algorithm and fault diagnosis algorithm.

\subsection{Soil moisture sensor suitable for WSN}

Soil moisture sensor, an important part of the wireless sensor network node, which has the following constrains.

a) Power energy is limited. The nodes are very small which just equipped with alkaline battery as energy source and replacing batteries in time is infeasible, which requires that the system must work in low power 
dissipation and so as the sensor. According to the application requirement, continuous working current of sensor must under $0.5 \mathrm{~mA}$ in our system. (Blumberg et al., 2001; Li et al., 1999)

b)Finite volume. In general, micro size is a greater advantage of WSNbased technology and bigger size would destroy the environment of the field, so the size of sensor is expected the smaller the better. Ideal size above ground is smaller than 200cm3. (Ma et al., 1993; Wang et al., 1991)

c) Finite computing and storage capability of WSN node result into that the sensor output of result must short. Too complex result certainly will cost the microprocessor more time and power. An 8 bits binary result or $0 \sim 3 \mathrm{~V}$ analog signals are proper.

d) The sensor must have flexible work mode and highly measuring speed. The node works alternatively with sleep mode and mode awake, most time being in sleep. Sensor requires the ability of adjust the working mode and output the sampling result immediately, so as that the WSN nodes can go into sleep as quickly as possible to save as much energy as possible.

Based on above conditions, ECH2O soil sensor of Decagon Devices Company is selected in the system (Figure. 2).

The $\mathrm{ECH} 2 \mathrm{O}$ probe gets the volumetric water content by measuring the dielectric constant of the soil. Since the dielectric constant of water is much higher than others, that of water is 80 , soil minerals 4 , air 1 , the dielectric constant of the soil is a sensitive measure of water content. The ECH2O probe has a very low power requirement and high resolution which gives us the ability to make as many measurements as we want over a long period of time, with minimal battery usage. (Hu et al., 2004; Luo et al., 1990)

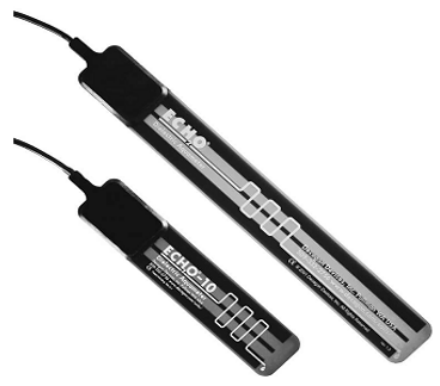

Figure 2 Photo of ECH2O

\subsection{Covering algorithm based on time and space concept}

Coverage is an important part of the WSN technology. As application oriented, definitions of WSN coverage is different from each other. Aiming at cotton field soil moisture monitoring, single coverage is defined as that for a certain region in the field, its soil moisture can be monitored by at least one node (Mao et al., 2007; Ren et al., 2006) .So does it for k-times repetitive 
coverage, which is that soil moisture can be monitored by at least $\mathrm{k}$ nodes for certain region in the field. Considering the features of soil moisture monitoring and reducing the energy consumption, single coverage is concerned in the following only. (Zhang et al., 2005; Wang et al., 2007)

Coverage in plane space has many mature algorithms and models, such as Boolean sensing model, grid deployment model and so on. Best deployment in certain time can be got through one model. Although the growing of cotton plant has influence on the communication distance between nodes, researching covering algorithm more deeply and finding an algorithm which can utilize the energy furthest but not just by adding redundant nodes become an difficult problem. ( Xu et al 2007)

Besides, the following are also what should be studied in this part:

a) Algorithm of initial deployment used in cotton field, to find an easy and rapid method for implementation;

b) How to guarantee the quality of data from the important area. Kcoverage can be considered if needed.

\subsection{Election of cluster head/ cluster building and fault diagnosis algorithms}

\subsubsection{Election of cluster head/ cluster building}

Because that the topology is based on plane space and time, the cluster head and cluster change as time elapsing and cotton growing. Whether election of cluster head and cluster building is reasonable or not can directly affect the quality of service of network. A reasonable routing table and a rapid election algorithm is the key for solving the upper problem.

\subsubsection{Health diagnosis algorithms}

WSN-based soil moisture monitoring field network has the feature of large mount of nodes, so it is difficult and impossible to maintain it manually. It requires that the node has nice noise tolerance and equips with nice fault diagnosis algorithm in order that the node can adjust itself as timely as possible.

The feature for the diagnosis algorithm is its statistical calculation, or based on entropy (Wang et al., 2004; Zhang et al 2004). Because nodes are exposed in open air and suffered from corrosion, high-low temperature and restricted energy, that the node gets out of order accidentally is very normal. Diagnosis algorithm must have the ability to distinguish accidental fault from frequent fault. One way to solve this is that diagnosis algorithm judges 
the accident and counts its occurring time and then the diagnosis algorithm estimates the state of the node by using statistics. What needs to further study is that how to distinguish the fault before the statistic model is established, how to identify the wrong data and how to reduce the work time of diagnosis algorithm to adjust the node as quickly as possible.

Further more, stub-network is also one part of diagnosis. Paths by which sensor node data is transferred to sink node are expected to distribute in several different ways statistically, for prevention that part nodes would die in advance. If data from one node or one cluster is delayed in only way, the cluster are called stub-network. Stub-network is not allowed to appear in the network. Building a diagnosis for the sub-work and locate its position are need.

\section{EXPERIMENTAL STUDY}

The studying team preliminary structures soil moisture monitoring platform in National Precision Agriculture Demonstration Bases in Beijing for the long-term experiments. In order to facilitate the analysis of statistics, server software was developed. The server can receive sensor nodes data, and playback of each sensor node dynamic data transmission path.

Figure2 shows distribution of sensor network. Experiment was carried on in a cotton field with 5000 meter long and 2000 meter width.

The diagnosis algorithm and coverage algorithm experiment are further developed on the platform, and the exhausted nodes are found and alarm. The soil moisture monitoring system is improved.

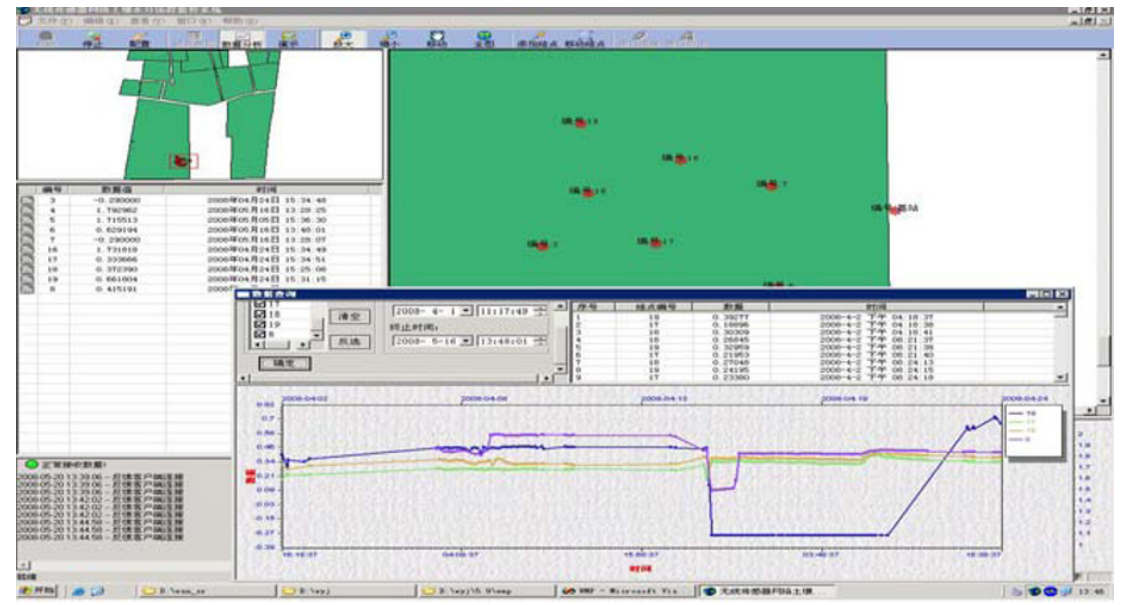

Figure 3 Photo of PC analysis software 


\section{CONCLUSION}

Wireless sensor networks has unparalleled advantage to traditional technologies in soil moisture monitoring. The article shows the framework of WSN application in cotton soil moisture monitor system. Combining with experiments, thinking on how to solve the technical difficulties is also been shown in the article. Which worth to be emphasized is that the system can also be used in other application by just a little alteration, such as temperature and humidity monitor in field, monitoring in wheat field and so on.

\section{ACKNOWLEDGEMENTS}

This work was supported by a grant from Key Technology R\&D Program of China (No. 2007BAD44B06).

\section{REFERENCES}

Blumberg DG, Freilikher V (2001), "Soil water-content and surface roughness retrieval using ERS-2 SAR data in the Negev Desert", Israel. J. Arid Environ. 49:449-464;

Chen Z, Zhou Q, Tang H (2004), "The future agricultural applications demanding on spaceborn remote sensing in China”, In: H Tang (ed) Research on the Agricultural Resource Utilization and Regional Sustainable Development. China Outlook Press, Beijing, pp 236250 ;

Hu Jiandong, Duan Tiecheng, He Sailing, "Study of a Capacitance-Based Self ModelEstablishing Sensor for Measuring Water Content of Soil", Journal of Transcluction Technology, 1st, 2004;

Jing Qi, Chen Zhoufeng, Guan Zhi, Wang Zhao, "Design and deployment of wireless sensor networks", Computer Engineering and Applications, 21st, 2007;

Li J, Islam S (1999), "On the estimation of soil moisture profile and surface fluxes partitioning from sequential assimilation of surface layer soil moisture". J. Hydrol. 220:86103;

Luo Rufang, Lou Guichang, LuYuping, Yian Ronhua, "Study of the Si-MOS Capacitive Type Micro Humidity Sensor", Journal of Transcluction Technology, 3rd, 1990;

Ma Xiaoyi, Xiong Yunzhang, Sun Mingqin, “The Development of Capacity Soil Moisture Sensor", Journal of Transducer Technology, 1st, 1993;

Mao Yingchi, Feng Guofu, Chen Lijun; Chen Daoxu, "A Location-Independent Connected Coverage Protocol for Wireless Sensor Networks", Journal of Software, 7th, 2007;

Ren Yan, Zhang Sidong, Zhang Hongke, "Theories and Algorithms of Coverage Control for Wireless Sensor Networks", Journal of Software, 3rd, 2006;

Wang Chao, Wang Hailing, Shi Xiangyong, Gong Xu, "Routing Algorithm for WSN Based on Swarm Intelligence Optimization", Journal of Shanghai University (Natural Science Edition), 4th, 2007; 
Wang Gang, Zhao Hai, Wei Shouzhi, "Decision-Level Fusion Model Based on Threat Game Theory", Journal of Northeastern University (Natural Science), 1st, 2004;

Wang wei, Qi Changyong, "Soil-moisture Sensors", Journal of Transducer Technology, 2nd, 1991;

Xu Congfu, Li Shijian, Rao Dazhan, Pan Yunhe, "Triangle-Arranged Method for Coverage with Connectivity in Sensor Networks", Chinese Journal of Sensors and Actuators, 3rd 2007;

Zhang Qing, Xie Zhipeng, Ling Bo, Sun Weiwei, Shi Baile, "A Maximum Lifetime Data Gathering Algorithm for Wireless Sensor Networks", Journal of Software, 11th, 2005;

Zhang Xiaodan, Zhao Hai, Wang Gang, Wei Shouzhi, "Fusion Algorithm for Uncertain Information by Fuzzy Decision Tree", Journal of Northeastern University (Natural Science), 7th , 2004; 